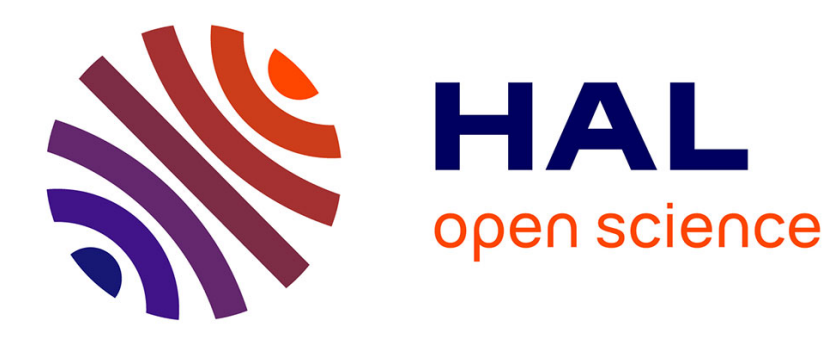

\title{
La Madeleine et le Panthéon, destins croisés de deux monuments parisiens
}

\author{
Albert Levy
}

\section{To cite this version:}

Albert Levy. La Madeleine et le Panthéon, destins croisés de deux monuments parisiens. Les Annales de la Recherche Urbaine, 2004, 96, pp.157-163. halshs-00124629

\section{HAL Id: halshs-00124629 \\ https://shs.hal.science/halshs-00124629}

Submitted on 23 Feb 2007

HAL is a multi-disciplinary open access archive for the deposit and dissemination of scientific research documents, whether they are published or not. The documents may come from teaching and research institutions in France or abroad, or from public or private research centers.
L'archive ouverte pluridisciplinaire HAL, est destinée au dépôt et à la diffusion de documents scientifiques de niveau recherche, publiés ou non, émanant des établissements d'enseignement et de recherche français ou étrangers, des laboratoires publics ou privés. 


\section{La Madeleine et le Panthéon : destin croisé de deux monuments parisiens Malaise dans la transmission}

Le religieux et le politique contribuent, à travers leurs conflits et leurs connivences, à l'édification des sociétés : par leurs confrontations ils participent à la fabrication du lien social et à la construction de l'identité collective. Les rapports d'intrication / désintrication entre ces deux forces qui se sont combattues au cours de l'histoire, l'une voulant dominer l'autre, avec aussi parfois des moments de collusion et de complicité plus ou moins forts, se traduisent dans l'espace à travers des formes et des langages stylistiquement codés et datés : on peut lire l'histoire mouvementée de ces relations inscrites dans le territoire urbain.

Les grands monuments - c'est un lieu commun - sont des pétrifications de l'histoire d'une nation. Ils sont le condensé de grands événements survenus à une collectivité, et restent, à ce titre, des témoignages matériels de ce passé : on peut décrypter à travers eux ces moments-clés qui ont forgé l'identité d'une nation et dessiné le visage de son espace, on peut y lire, comme dans les pages d'un livre, son passé, saisir son évolution, ce qui constitue sa particularité. Ils rendent compte, à travers leur conception, leur réalisation, leur usage et leur transformation, des événements et des conflits majeurs qui ont traversé et construit une société. Ils sont aussi des moments de synthèse où se sont cristallisées les idées esthétiques et où se sont matérialisés les mouvements artistiques, reflets d'une époque et de sa sensibilité. Tels des traces, des dépôts laissés par le cours sinueux de l'histoire, indices de son passage, les monuments témoignent de ce passé qui a forgé l'identité d'une société.

Entre l'intrication totale des deux forces du politique et du religieux entraînant leur confusion (intégrisme, Ancien Régime, sociétés islamiques...), ou leur désintrication complète, c'est-à-dire leur stricte séparation qui peut être neutre (toutes les religions sont tolérées, sans parti pris ni privilège), ou partiale (soit l'Etat défend l'athéisme et interdit le religieux comme dans les régimes totalitaires, soit il soutient la laïcité en isolant le religieux dans le privé), entre ces deux positions extrêmes, on trouve une gamme de combinaisons possibles, donc de désintrication partielle et variable de ces deux forces, résultant à la fois de son degré de développement, mais aussi du rapport particulier, et parfois ambigu, entretenu avec le religieux dans tel ou tel pays, à telle ou telle période de son histoire. Cette distribution géographique des différents rapports d'intrication /désintrication entre ces deux forces qu'on peut observer à un moment donné, selon les pays, peut également s'observer dans l'histoire même d'un pays à travers, cette fois, l'évolution de ces rapports dans le temps, dans leur alternance diachronique, résultant des diverses relations contractuelles ou polémiques que ces forces ont entretenues.

C'est de la fluctuation de ces rapports d'intrication /désintrication entre les deux forces dont il sera question ici, à travers le cas de deux monuments parisiens bien connus, le Panthéon et la Madeleine, contemporains dans leur origine (les deux projets sont dus à l'initiative de Louis $\mathrm{XV}$ ). Ils connaîtront un destin croisé et symétriquement inverse : en gros, le premier, sur la rive gauche, a commencé sa carrière comme église et l'a fini comme temple civique, le second, sur la rive droite, a démarré son existence comme temple civique et militaire et l'a terminé comme église. Ces deux monuments ont connu une évolution mouvementée, résultat de l'instabilité politique de cette période très tourmentée de l'histoire de France qui va du milieu du XVIIIème à la fin du XIXème qui a connu pas moins de dix régimes successifs au cours desquels le religieux et le politique 
se sont affrontés laissant chaque fois des traces et des marques de cet affrontement sur nos deux édifices : ce qui se jouait dans cette période c'était l'avenir de la Révolution de 1789 .

Les rapports d'intrication / désintrication entre politique et religieux vont en effet alterner au cours de ces régimes successifs, se traduisant chaque fois par un impact particulier sur les deux monuments, tant sur le plan de son usage que sur celui de sa forme. Telle une (re)écriture périodique et répétée sur un même document, ces dépôts successifs de traces et de signes dans le temps feront de ces deux monuments de véritables palimpsestes. Après avoir présenté les deux cas, leur rôle dans la forme urbaine et la continuité urbanistique qui caractérisent les projets, nous montrerons l'impact de ces rapports d'intrication / désintrication, de ces fluctuations du rapport entre politique et religieux à travers l'histoire, sur ces deux édifices, au cours des régimes successifs qu'ils ont traversés et qui ont laissé leurs empreintes. Nous nous interrogerons, en conclusion, sur leur évolution actuelle et sur leur devenir.

\section{Panthéon, Madeleine: continuité urbanistique des projets dans le temps}

Sur le plan urbanistique, les deux projets conçus à la fin du XVIIÌme siècle, relèvent des mêmes procédés de composition urbaine de cette période néo-classique ${ }^{1}$ : "insularisation" du monument au milieu d'une place ${ }^{2}$, symétrisation et régularisation de l'édifice, mise en perspective du monument par dégagement d'un axe visuel sur sa façade principale (technique dite du "monument-cible"). Cet axe visuel est aussi un axe de composition contrôlant et organisant l'espace urbain environnant. Tous ces procédés pour isoler le majeur sont destinés à accentuer la monumentalité. Le monument est conçu à la fois comme un nœud et comme un repère dans la ville : comme nœud, il articule un réseau de relations avec d'autres monuments et édicules urbains importants dans un environnement plus ou moins large; comme repère visuel, il identifie et localise un quartier dans la ville auquel il donne alors son nom

Les deux projets sont dus à l'initiative de Louis XV (1710-1774), mais à partir de deux démarches, deux chronologies inverses : l'un, celui de Madeleine, part d'une place et d'un aménagement externe assez vaste, pour aller ensuite vers la réalisation de l'édifice, l'autre, celui du Panthéon, part de la conception de l'édifice pour s'élargir vers la réalisation de la place et de tout l'aménagement environnant. Nous allons rapidement présenter, dans un premier temps, les grandes étapes de réalisation de ces deux projets, pour étudier, dans un second temps, l'impact des deux forces - plus exactement leurs rapports d'intrication /désintrication en fonction des régimes traversés - sur le destin de ces monuments.

Pour la Madeleine le processus est inverse : c'est l'aménagement extérieur qui va déterminer l'implantation et la localisation de la future église. Le point de départ est, en effet, l'aménagement de la Place Louis $\mathrm{XV}^{3}$ (future Place de la Concorde). En 1750 le

\footnotetext{
${ }^{1}$ Sur l'architecture classique, L Hautecoeur, Histoire de l'architecture classique en France, t. IV, V, VI, Paris, 1953

${ }^{2}$ Architecte de la fin du XVIIème siècle, L Savot, L'architecture française des bâtiments particuliers, seconde édition, Paris, 1865, écrivait :"Les nobles bâtiments ... devraient être isolés, c'est-à-dire détachés, et séparés des autres de toutes parts...". Sur cette question J Lucan, Les Cahiers de la recherche architecturale $\mathrm{n}^{\circ} 5$, mars 1980 , Isoler le majeur, p 35-40.

${ }^{3}$ B de Montgolfier, La place Louis XV, Monuments historiques $\mathrm{n}^{\circ} 120$, avril 1982 ; A Mousset, op. cit.
} 
prévôt de Paris veut offrir une statue équestre au roi qui propose, pour l'installer, un terrain lui appartenant situé entre les Tuileries et les Champs Elysées ; mais il faut auparavant l'aménager. Après un concours, le roi choisit Gabriel qui réalise la place en 1755. La statue équestre de Louis XV par Bouchardon est élevée en son centre en 1763. C'est une place royale totalement inédite par rapport à la tradition des places royales connues : entourée de fossés, avec des guérites aux quatre coins, fermée seulement au Nord par deux bâtiments symétriques de part et d'autre de la rue royale située dans l'axe de la place, elle est complètement ouverte sur les trois autres cotés. Comme beaucoup d'espaces majeurs de la capitale, elle subira, elle aussi, des changements de forme et de dénomination, elle sera surtout, à la Révolution, le théâtre de la décapitation de Louis XVI (1793). Rappeler ou effacer ce sinistre souvenir, c'est la tache que vont s'efforcer d'accomplir les régimes suivants en fonction de leur orientation politique.

Simultanément en 1757, Louis XV décide de la construction d'une nouvelle église de la Madeleine ${ }^{4}$, en remplacement de l'ancienne devenue trop petite par rapport au développement du faubourg Saint-Honoré, et propose de la situer dans l'axe de la nouvelle rue royale qui donne sur la place. Il confie le projet à Contant d'Yvry qui proposa un plan en croix latine avec un péristyle surmontée d'une coupole. A sa mort en 1777, Couture modifie le projet en élevant un portique et en supprimant le péristyle : la Madeleine aurait été alors sur la rive droite le pendant de l'église Sainte-Geneviève sur la rive gauche. Mais la construction de l'église est ralentie, sinon bloquée, par les événements et les changements rapides de régimes qui vont avoir lieu. L'aménagement extérieur va cependant se poursuivre : le pont de la Concorde est réalisé dans la continuité de l'axe par Perronet en 1791, et toujours dans le prolongement de cet axe, en contre-point à la façade de la Madeleine (non encore finie), Poyet élève le portique de l'Assemblée nationale (ancien palais Bourbon) en 1806, organisant ainsi une sorte de face à face entre le politique et le religieux de part et d'autre de la place. Cet axe sera renforcé par les nouveaux aménagements de la place conçus par Hittorff entre 18301835 qui réalisera, de chaque coté de l'obélisque de Louxor planté au centre, deux fontaines. Des statues allégoriques de huit grandes villes françaises seront posées sur les guérites, et des colonnes rostrales encadreront aussi la place. Haussmann comblera finalement les fossés pour lui donner son allure actuelle. La place de la Concorde devient un lieu de réconciliation nationale, vide de tout symbole politique partisan, un lieu neutre connotant uniquement l'unité territoriale nationale (ses fleuves, ses mers, ses villes). L'église est terminée en 1842 par Huvé qui poursuit l'œuvre de Vignon disparu en 1828. Il faudra attendre la percée du Bd Malesherbes sous le Second Empire, symétriquement au $\mathrm{Bd}$ de la Madeleine, pour donner à la place de la Madeleine la configuration finale que nous connaissons.

Cet axe Nord-Sud va croiser au centre de la Place de la Concorde l'axe Est-Ouest, dit aussi axe majeur, ou axe historique, qui part du Louvre à partir de l'arc du Carrousel (1808) - son point de départ véritable - pour traverser le Jardin des Tuileries conçu par Le Notre qui souligne cet axe dans son dessin, pour aller jusqu' à l'arc de Triomphe, en orientant le tracé des Champs Elysées. Aujourd'hui cet axe s'étire jusqu'à la Défense (Grande Arche) et, au-delà, sur plusieurs kilomètres, afin de restructurer la banlieue Ouest (Nanterre). On mesure l'importance capitale de ces deux axes perpendiculaires pour l'aménagement et la composition de tout l'Ouest de la capitale, la Madeleine occupant un des points de structuration de ces deux axes, l'axe Nord-Sud. Le projet de la Madeleine est donc indissociable de l'aménagement global de l'espace environnant, ici assez vaste. Là encore, on constate une continuité dans la conception urbanistique, dans

\footnotetext{
${ }^{4}$ A Krugier, La Madeleine, Desclée de Brouwer, Paris, 1937; A Mousset, op.cit.
} 
la composition de la forme urbaine, malgré les fréquents changements d'usage de l'édifice en fonction des régimes politiques successifs.

\section{Panthéon, Madeleine : fluctuation des projets dans le temps}

Prés de dix régimes vont se succéder entre le milieu du XVIIème et la fin du XIXème siècle durant lesquels on examinera, plus particulièrement, les rapports du politique au religieux et l'impact que ces rapports ont eu sur les projets des deux monuments, sur les fluctuations de leur forme et de leur destination. On peut rapidement rappeler ces régimes : 1-Ancien Régime (.. -1789), 2- Révolution et Ière République (1789-1792 et 1792-1804), 3-Ier Empire (1804-1814), 4-Restauration, Louis XVIII (1814-1824) et Charles X (1824-1830), 5-Monarchie de Juillet, Louis-Philippe (18301848), 6-Ilème République (1848-1852), 7-Ilème Empire (1852-1870), 8-IIIème République (1870-1940). Cette liste de régimes n'est que la traduction d'une longue et pénible marche vers la consolidation des acquis de la Révolution. Comme l'a bien vu $\mathrm{F}$ Furet $^{5}$, avec la IIIème République la Révolution se termine après au moins un siècle de luttes pour réaliser ses objectifs, les rapports entre les deux forces vont alors se stabiliser pour un moment. Nous allons donc passer en revue ces différents régimes successifs pour y examiner la nature des rapports entre le politique et le religieux, et leurs impacts sur les deux monuments.

\section{1) Ancien Régime (...-1789) : intrication totale}

C'est une période de collusion et de complicité parfaite entre le pouvoir religieux et le pouvoir politique qui s'appuient l'un sur l'autre, on peut parler ici d'intrication totale des deux forces : la monarchie est d'origine divine. Cette collusion a pris une tournure particulière en France, sous le nom de gallicanisme, pour affranchir l'Eglise de France du Saint-Siège. Le pluralisme religieux y est inconcevable selon le principe Cujus regio, ejus religio, ou tel prince, telle religion, engendrant persécution, expulsion et intolérance vis-à-vis de tout ce qui n'est pas catholique.

C'est dans ce contexte d'intrication totale des forces que Louis XV, chef de l'Eglise de France, décide de la construction de deux églises à Paris : Sainte-Geneviève (1744) sur la rive gauche, et la Madeleine (1757) sur la rive droite.

2) Révolution et Ière République (1789-1804) : désintrication complète

C'est une période de rupture entre le pouvoir politique et le pouvoir religieux marquée par les exactions des révolutionnaires contre les ecclésiastiques, la confiscation des biens religieux, l'expulsion des Ordres... On peut parler à présent de désintrication complète des deux forces. La religion catholique cesse de devenir religion dominante, religion d'Etat, elle est mise à égalité avec les autres, la séparation de l'Eglise et de l'Etat (1795) introduit la laïcité en reléguant la religion à une affaire privée. Dans ce contexte de désintrication les deux projets vont subir des modifications substantielles.

- L'église Sainte-Geneviève est par décret de 1791 transformée en Panthéon destiné à recevoir les cendres des grands hommes de la Nation: Mirabeau, Voltaire, Rousseau, Marat $^{6} .$. Quatremère de Quincy est chargé de transformer l'édifice de Soufflot: suppression des clochers et de la lanterne, changement du programme décoratif religieux, notamment sur le portique, obturation des 45 baies pour créer une obscurité

\footnotetext{
${ }^{5}$ F Furet, La Révolution 1770-1880, Hachette, Paris, 1988

${ }^{6}$ Mirabeau et Marat en seront vite chassés
} 
propice à la méditation... morale ; l'église se mue en un vaste mausolée et la crypte en nécropole. Sur le frontispice on enlève l'inscription latine dédiée à sainte Geneviève et on la remplace par Aux grands hommes la patrie reconnaissante, sur le fronton le décor religieux est supprimé pour un décor laïc, et sur le dôme on change la croix par une statue de la Renommée.

- A la place de l'église de la Madeleine, dont la réalisation à peine commencée est suspendue, on envisage une succession de projets alternatifs sur ce lieu : une salle d'Assemblée nationale, puis un temple à la Révolution à proximité de la guillotine, une salle des Commémorations nationales, une Banque de France, une Bibliothèque, une Bourse, un Opéra, un Tribunal de commerce... aucun projet ne verra le jour.

\section{3) Empire (1804-1814) : désintrication partielle}

Après la rupture radicale opérée par la Révolution, Napoléon va s'employer à rétablir les liens avec l'Eglise en signant le Concordat de 1801, ce compromis rétablit une nouvelle forme de gallicanisme. Le débat va se faire maintenant entre ultramontanisme (partisans du pouvoir du Saint-Siège) et gallicanisme (autonomie par rapport au Saint-Siège). L'Empire cherche cependant à s'appuyer sur l'Eglise, Napoléon se fait sacrer par le pape Pie VII en 1804. Il organise les autres religions (protestantisme et judaïsme) en consistoires pour pouvoir également mieux les contrôler. C'est dans ce contexte de désintrication partielle que les deux édifices vont recevoir des destinations nouvelles.

-Le Panthéon est restitué au culte catholique dans sa partie haute en 1806, sa partie basse, la crypte, est affectée à l'inhumation des grands serviteurs de l'Etat. Avec ce compromis il devient à la fois une église et un temple (laïc).

-A l'emplacement de la Madeleine et à la place de l'église prévue, Napoléon décide également en 1806 de construire un temple à la gloire de la Grande Armée, et impose, à la suite d'un concours, le projet de Vignon - un temple gréco-romain- au jury qui avait choisi Beaumont en justifiant son choix : "c'est un temple que j'avais demandé et non une église...Par temple j'ai entendu un monument tel qu'il y en avait à Athènes et qu'il n'y en a pas à Paris."

\section{4) Restauration (1814-1830) : intrication totale}

Avec le retour des Bourbons c'est à un quasi rétablissement de l'Ancien Régime auquel on assiste (le drapeau tricolore et la Marseillaise sont supprimés), retour à la monarchie de droit divin. Le catholicisme redevient religion d'Etat, religion dominante, et les persécutions des protestants reprennent dans le midi par exemple (Terreur Blanche 1921-27). C'est une période de renouveau pour l'Eglise avec une hausse des ordinations, un retour des Ordres religieux (Jésuites, Trappistes, Dominicains...), et les idées ultramontaines triomphent (modèle catholique italien et pouvoir du Vatican). On peut parler dans ce nouveau contexte d'un retour à l'intrication totale des deux forces avec de nouvelles conséquences pour les deux édifices.

-Le Panthéon est entièrement réaffecté au culte catholique et redevient église Sainte Geneviève. La présence des sarcophages de Rousseau et de Voltaire n'est plus tolérée, on veut les expulser de l'église ; ils sont finalement cachés dans la crypte dans un caveau fermé et à l'écart (sous le portique), non visibles. Le décor de l'église reprend avec une fresque peinte sous le dôme, l'Apothéose de sainte Geneviève (1827). A l'extérieur, sur le frontispice l'inscription, Aux Grands Hommes..., est supprimée remplacée par celle latine dédiée à sainte Geneviève, sur le fronton une croix avec des rayons est resculptée, et enfin sur le dôme une croix en bronze est reposée. 
-Pour la Madeleine en construction, Louis XVIII décide, dans un premier temps, d'en faire une église expiatoire à la mémoire de Louis XVI et de Marie-Antoinette, face à la place où s'est déroulée leur exécution. Vignon est chargé de réaliser ce nouveau programme, mais décédé en 1928, Huvé reprend son projet et poursuit le chantier qui connaît de graves difficultés financières qui le ralentissent. Entre temps une chapelle expiatoire est construite un peu plus loin, en 1826, sur l'emplacement du cimetière de la Madeleine où avaient été inhumés, parmi les 1300 guillotinés, les corps du roi et de la reine

5) Monarchie de Juillet (1830-1848) : désintrication partielle

La révolution de 1830 chasse les Bourbons, Louis-Philippe d'Orléans, cousin du roi Charles $\mathrm{X}$ est installé. Le drapeau tricolore est restauré, l'idée de religion dominante abandonnée, la nouvelle monarchie réalise un compromis : on assiste à une période de désintrication partielle entre les deux forces qui se traduit par de nouvelles décisions pour les deux monuments.

-L'église Sainte-Geneviève redevient Panthéon, le décor laïc intérieur sur les pendentifs est peint représentant la Gloire, la Mort, la Patrie, la Justice (1837). A l'extérieur le fronton reçoit en 1837 son ornement actuel, la Patrie décernant ses récompenses, sur le frontispice l'inscription, Aux grands hommes..., est rétablie, et un drapeau tricolore flotte à la place de la croix au sommet du dôme.

-Pour la Madeleine Louis-Philippe prévoit d'abord d'en faire un sanctuaire de la réconciliation nationale puis se rétracte. Le projet avance grâce au concours financier de l'Etat : les voûtes sont achevées en 1831, le décor du fronton est taillé en 1833, la grille et le dallage extérieurs sont terminés en 1835, la fresque intérieure dans l'abside, Histoire du christianisme (ou trône au centre Napoléon), est peinte entre 1835-37 ainsi que les sculptures du maître-autel, le Ravissement de Madeleine, et en 1841 les portes d'entrée en bronze, où sont représentés les Dix commandements, sont achevées...L'église est terminée et inaugurée en 1842, après plus de 90 ans de vicissitudes. L'Etat, qui a supporté tous les frais, remet le bâtiment à la ville, il est consacré église paroissiale en 1845 .

6) Ilème République (1848-1852) : désintrication partielle

La Révolution de 1848 chasse Louis-Philippe et la monarchie. Fragile et éphémère la Ilème République cherche une entente avec le catholicisme : l'idée de séparation de l'Eglise et de l'Etat dans la liberté réciproque voit le jour, cependant que les lois Falloux de 1850 permettent à l'église de développer un enseignement confessionnel à tous les niveaux. On voit donc se poursuivre durant cette courte période une désintrication partielle des deux forces.

-Dans sa ferveur lyrique la IIème République veut faire du Panthéon un temple à l'humanité

-La Madeleine, qui entame sa carrière d'église paroissiale poursuit son décor intérieur (peintures et sculptures religieuses...)

7) Ilème Empire (1852-1870) : intrication totale

Le coup d'Etat de napoléon III ramène un régime où le politique et le religieux vont entretenir une nouvelle collusion complète. L'Empire est vécu comme une victoire, une revanche de l'Eglise contre les révolutionnaires, comme une nouvelle alliance du trône et de l'autel, avec même plus de moyens que durant la Restauration. Cette nouvelle complicité des deux forces se traduit, par exemple, par une invasion de l'espace public 
par la statuaire religieuse : comme l'écrit $\mathrm{M}$ Agulhon ${ }^{7}$ on assiste, dans cette période, à l'opposition mariolâtrie (culte de Marie) contre mariannolâtrie (culte de Marianne), à une "reconquête des âmes (par) l'entreprise proprement visuelle de christianisation du décor de la vie". On retombe dans un contexte d'intrication totale avec des conséquences sur les deux monuments.

- Napoléon III restitue le Panthéon au culte catholique qui redevient église SainteGeneviève. A l'extérieur on supprime sur le frontispice l'inscription Aux grands hommes... pour remettre la formule latine de départ, si sur le fronton le décor reste inchangé, sur le dôme, en revanche, une croix est reposée.

- La Madeleine confirmée dans sa fonction d'église paroissiale poursuit son décor iconographique.

8) IIIème République (1870-1940) : désintrication complète

La Commune écrasée, et après une courte période de pseudo République avec les deux gouvernements de l'Ordre moral (Thiers, Mac-Mahon) où se poursuit l'étroite association entre Eglise et pouvoir public, une réaction violente s'opère à partir de 1880 ("Le cléricalisme, voilà l'ennemie", Gambetta) : sécularisation de la vie sociale et culturelle, loi contre les congrégations et les Ordres religieux, laïcisation de l'enseignement public, loi de séparation de 1905. D'autres mesures vont toucher également la vie publique comme la déconfessionnalisation des cimetières, l'interdiction des emblèmes religieux dans les hôpitaux, dans les tribunaux, la prohibition des processions religieuses sur la voie publique, le service militaire obligatoire pour les séminaristes...Cette offensive contre le pouvoir religieux conduit à une situation de désintrication totale entre les deux forces, et, dans ce contexte, les deux édifices vont connaître une nouvelle affectation qui semble définitive ${ }^{8}$.

- L'église Sainte-Geneviève, en fonction jusqu'en 1876, redevient Panthéon et accueille triomphalement les cendres de V Hugo en 1885. Depuis, de nombreux hommes politiques, écrivains, scientifiques, résistants, ... sont honorés en accédant au temple, et viennent côtoyer les généraux de l'Empire : E Zola, J Jaurès, F Eboué, V Schoelcher, J Moulin, R Cassin, J Monnet, P et M Curie, A Malraux.....En 1874, les murs de l'église reçoivent encore des décors de peinture dédiée à l'histoire de sainte Geneviève et à l'épopée des origines chrétiennes et monarchiques de la France. C'est entre 1912-1924 que le Panthéon reçoit sa sculpture laïque qui est placée contre les 4 piliers du dôme (Aux orateurs et publicistes de la Restauration, A Diderot et aux encyclopédistes, A J J Rousseau, A la gloire des généraux de la Révolution) et dans l'ex-chœur on installe un groupe imposant, la Convention nationale. A l'extérieur sur le frontispice, on réinscrit Aux grands hommes..., sur le fronton le décor, La patrie décernant les récompenses, est toujours là, mais sur le dôme, paradoxalement, on conserve la croix du Second Empire. - La Madeleine poursuit sa carrière d'église paroissiale et continue d'aménager son décor interne avec une mosaïque dans l'abside (1893).

\section{Malaise dans la transmission : quel avenir pour les deux monuments ?}

\footnotetext{
${ }^{7} \mathrm{M}$ Agulhon, Marianne au combat, L'imagerie et la symbolique républicaine de 1789 à 1880 , Flammarion, Paris, 1979

${ }^{8}$ Le régime de Vichy (1940-44) revient cependant sur cette désintrication : la Révolution nationale de Pétain est antilaïque. Pour l'Eglise la défaite de 40 est due à l'impiété de la IIIème République, à l'apostasie nationale, cause de la décadence de la nation, la sécularisation l'a mis en danger de mort.
} 
On a vu comment les avatars de ces deux monuments, à travers l'histoire, a suivi les vicissitudes des différents régimes qui se sont succédés. Cependant leur implantation, et la composition urbaine qu'ils ont induite, sont bien restées inchangées malgré ces fluctuations des projets : ils ont été, et restent, des éléments forts et structurants de l'espace urbain. On peut se demander ce que deviennent actuellement ces édifices dans la ville ? Quelle est la portée de leur message aujourd'hui, son impact ? Quel est l'usage qui en est fait? Quel avenir enfin pour ces deux monuments ?

Le Panthéon, temple paradoxal, est un édifice à plan centré qui a été conçu pour recevoir la châsse de sainte Geneviève en son centre. Plus qu'un temple, c'est aujourd'hui un mausolée, une nécropole pour morts illustres et célèbres : le haut de l'édifice fonctionne comme une sorte de gigantesque hall, une salle des pas perdus qu'on traverse pour se rendre en bas, dans la crypte, pour visiter les tombeaux. La Madeleine, église paradoxale, édifice de forme gréco-romaine, est un bâtiment religieux auquel on reproche parfois son manque de lumière intérieure et surtout son absence de signe chrétien extérieur (croix, clocher...). Comme l'écrit très justement A Mousset : " Tandis que le Panthéon laïcisé a gardé sa croix, la Madeleine est restée-selon le vou de Napoléon- 'un temple et non une église'. Pour mieux dire, le Panthéon est un monument païen sur un plan d'église, la Madeleine une église sur un plan païen." . On ne peut mieux décrire le croisement des deux monuments.

Dans son analyse du Panthéon comme lieu de mémoire, M Ozouf ${ }^{10}$ conclue par "l'échec du Panthéon", et de son culte : selon elle, il s'explique "dans la désaffection de l'image des grands hommes pour lesquels il a été aménagé...en ce sens la désuétude du Panthéon, malgré sa réanimation récente, c'est l'effacement des conditions intellectuelles et morales dans lesquelles il a été érigé...; elle précise plus loin cette crise de la transmission et ses causes : "La seconde raison tient au doute qui ronge désormais la croyance dans un art propagandiste et éducateur. Trop de réalismes socialistes ont discrédité la capacité de l'art à accomplir l'instruction et la réconciliation civique. Nous savons qu'on peut vivre au milieu de statues colossales et de peintures édifiantes sans les voir, et même en leur tournant le dos. Ce qui soutient le culte des grands hommes, au moment où il s'élabore, c'est la foi dans la solidarité spontanée de l'esthétique et de la morale, dans la nécessaire docilité du public à la leçon des sens et dans l'efficacité d'un art pédagogue. Nul ne peut croire aujourd'hui que la représentation visuelle soit le lieu même de la formation morale et de la manipulation idéologique et c'est en cela, aussi, l'échec du Panthéon."

Certes, M Ozouf a raison dans cette explication qu'elle donne de l'échec du Panthéon devenu, selon elle, lieu de mémoire morte. Mais il y a également d'autres causes à cet échec qui tiennent, selon nous, à la notion même de culte et à ses modalités de réalisation. Qu'est-ce qu'un culte et quelles sont ses conditions de fonctionnement? Dans un travail récent nous avons essayé de répondre à cette question en identifiant les conditions de fonctionnement d'un espace cultuel en général que nous avons défíni comme "machine à faire-croire"11, c'est-à-dire comme appareil de transmission et de persuasion (d'un récit, d'un message, de valeurs...). Une double médiation est requise pour l'existence d'un culte et de son espace : la première médiation instauratrice est constituée par les grands médiateurs (prophètes pour le récit religieux, "grands hommes" pour le récit républicain) fondateurs du culte, son message et de son contenu; la seconde

\footnotetext{
${ }^{9}$ A Mousset, op. cit. $\mathrm{p} 108$

${ }^{10}$ M Ozouf, Le Panthéon, L'Ecole normale des morts, in P Nora (éd), Les lieux de mémoire. I La République, Gallimard, Paris, 1984, p. 163

11 A Levy, Les machines à faire-croire, I Formes et Fonctionnements de la spatialité religieuse, Anthropos/Economica, Paris, 2003
} 
médiation commémoratrice est constituée par les médiateurs de second rang (prêtres dans un cas, "militants-officiants" diffuseurs des idées des fondateurs, dans l'autre), leur tâche consiste à sauvegarder et à transmettre le message, à le maintenir vivant en s'appuyant, pour cela, sur les ressources de l'art (inscrit dans l'espace, il doit relater, à travers l'iconographie, le récit de fondation des premiers médiateurs), ainsi que sur la liturgie (la célébration du message et des fondateurs).

La liturgie, en effet, récitation périodique, rappel incessant, temporellement rythmé et codifié, du récit et de son message, est également indispensable pour sa transmission. C'est donc cette deuxième médiation avec son espace cultuel, son personnel, sa liturgie, chargée de maintenir et de diffuser cette mémoire vivante, qui fait défaut ici, dans le temple du Panthéon, et dans son culte (en réalité absent), pour perpétuer le message républicain : il doit, en effet, pour rester une tradition, éviter de tomber dans l'Histoire en se transformant en archive, en mémoire morte. C'est donc l'absence de cette seconde médiation commémoratrice, le manque d'une réelle liturgie, la carence d'une éducation civique qui expliquent aussi l'échec du Panthéon et du message qu'il véhicule. Comment alors organiser un tel culte, quel contenu lui donner, quelle pratique de célébration organisée?

Concernant le devenir de la Madeleine, la crise tient à d'autres raisons, liée à la situation générale du catholicisme en Europe qui, sous l'effet de la sécularisation ${ }^{12}$ accélérée traverse une crise majeure marquée par la chute des vocations, du nombre de croyants, par la mutation des pratiques religieuses plus individualistes et éclectiques, les églises se vident, tendent à devenir des "coquilles vides", offrant un contraste saisissant entre la somptuosité, la grandeur de l'édifice, et la faiblesse de sa fréquentation, de son attraction, conduisant souvent à convertir l'espace à d'autres usages que religieux : la Madeleine n'y échappe pas.

On est donc là, face à cette évolution, devant deux cas de crise de la transmission ${ }^{13}$ auxquels nous avons essayé d'apporter un début d'hypothèses d'explication. Dans le premier cas (Panthéon), il s'agit d'une crise structurelle due à une défaillance de la seconde médiation commémoratrice (et de l'enseignement qui l'accompagne), dans le second cas (Madeleine), il s'agit d'une crise plus fondamentale, causée par une défiance vis-à-vis de la première médiation instauratrice, d'une mise en cause de la Révélation même, d'un problème lié à la foi. Si cette évolution se confirmait, elle poserait des questions sur le sens et l'avenir de ces deux monuments.

Albert Levy

\footnotetext{
${ }^{12}$ D Hervieu-Leger, Catholicisme, la fin d'un monde, Bayard Centurion, Paris, 2003 ; sur les mutations des pratiques religieuses, La religion pour mémoire, Cerf, Paris, 1993.

${ }_{13}$ Sur cette question lire R Debray, Malaise dans la transmission, Les Cahiers de médiologie, 11, Gallimard, avril 2001. Il fait de l'étude de la transmission, et de sa crise, l'objet central de la médiologie.
} 